\title{
Editorial
}

Francesco Asdrubali and Giovanni Brambilla

\section{Noise Mapping Special Issue: The noise climate at the time of SARS-CoV-2 Virus/COVID-19 Disease}

https://doi.org/10.1515/noise-2021-0015

Received Mar 30, 2021

When, about one year ago, this special issue was launched many countries were struggling to reduce the COVID-19 outbreak and its harmful effects. Such a large and rapid diffusion was never experienced in recent decades and strong changes have occurred in the people daily life due to the severe restrictions issued by the national Governments. These changes in the mobility and human behaviors have modified the acoustic environment, especially in urban areas, providing the opportunity to assess the corresponding modifications. For instance, sounds, such as natural ones, have been perceived during lockdown period as they were not masked anymore by transportation noise.

The response to the call for papers of this Special Issue was satisfactory and, after the peer-review process, 13 papers have been published in volumes 7 (2020) and 8 (2021), presenting experiences in different countries, also outside Europe.

The studies analyze the acoustic environment in many of its aspects: from the physical phenomenon to modelling its propagation and to estimate its impact, from its perception to its cultural identity and heritage.

The impact of mobility restriction on urban vehicular traffic and road noise pollution on the road network of Rome, Italy, is evaluated by Aletta et al. [1]. An overall noise reduction on the entire road network was found, even if its extent varied between road types. In a further analysis by Alsina Pagès et al. [2] carried out in some areas in Rome and Milan, Italy, traffic and non-traffic related noise events monitored from March to May 2020 are analyzed and compared to the corresponding values in 2019, in order to evaluate the effects of the lockdown period.

A similar approach was applied by Bartalucci et al. [3] to the noise data gathered by a smart noise monitoring system located in Monza, Italy. The changes occurred in noise levels trends during the lockdown period were compared with those collected by the same sensors in the same months of 2019. Some preliminary considerations regarding the reliability of the low-cost sensors are also reported. This type of analysis has been undertaken also by Zambon et al. [4] regarding the data gathered by the permanent noise monitoring system made of 24 noise sensors operating in Milan, Italy. An overall noise level reduction of about $6 \mathrm{~dB}$ was observed, leading to estimate an increase of $14.9 \%$ and $16.4 \%$ of the population respectively exposed to Lden below $53 \mathrm{~dB}(\mathrm{~A})$ and Lnight below $45 \mathrm{~dB}(\mathrm{~A})$ in comparison to 2019.

A study on additional features of the urban acoustic environment was presented by Aletta et al. [5] by the analysis of 30-second binaural recordings taken at 11 locations in London, representing a cross-section of urban public spaces with varying compositions of sound sources. Five acoustic and psychoacoustic metrics (LAeq, LA10, LA90, loudness, sharpness) were calculated for each recording and their changes from the pre-lockdown scenario (2019) to the lockdown scenario (2020) were investigated. Three types of urban settings were set considering their acoustic characteristics. An average reduction of $5.4 \mathrm{~dB}$ (LAeq) was observed, but

Francesco Asdrubali: Department of Engineering, Roma Tre University, Rome, Italy Giovanni Brambilla: CNR INM, Research Unit Acoustics and Sensors “O.M. Corbino”, Rome, Italy

๖ Open Access. ๑ 2021 F. Asdrubali and G. Brambilla, published by De Gruyter 
significant differences in the reduction were found across the locations, ranging from a $10.7 \mathrm{~dB}$ to a $1.2 \mathrm{~dB}$ reduction. The observed noise trends vary depending on the urban context.

The COVID-19 lockdown has provided the opportunity to study urban sound climate as never before and Manzano et al. [6] focused their investigation on some iconic sites in Granada, Spain, before (2019) and during the lockdown (2020). Results show a large change in environmental noise levels that is interesting not only because of its magnitude, but also for its implications, especially at those sites where social human activity was an identity feature. Natural or animal sounds became evident at some sites, especially where road traffic noise largely decreased, leading to significantly lower background noise levels. Important spectral changes were observed before and during the lockdown, suggesting a shift from anthropic to animal sources in the acoustic environment. Another study by De Lauro et al. [7] focused on the signature of the sound from the famous "Fontana di Trevi" in Rome, Italy, and recognized how it interacts with other features. The spectral soundprint obtained was different to the one determined in a time period far from the lockdown, the latter showing additional components in the $0.7-1 \mathrm{kHz}$ band frequency, which seems to act as a synchronization mechanism. The unique Covid-19 "silence" let emerge sounds that had been in the place for centuries, and offered the possibility to document them for preservation issues and cultural heritage.

The contribution by Munoz et al. [8] analyzes noise data from 21 continuous monitoring stations for the entire lockdown period in five conurbations in the south of France and compared them to the values in the "normal" situation (before the lockdown). Reductions from $4 \mathrm{~dB}$ to $6 \mathrm{~dB}$ (Lden) were observed for monitoring stations with highly dominant road noise and new noise maps for road noise, corresponding to the lockdown situation, were drawn for the conurbations of Lyon and Grenoble. The impact of such reductions in terms of noise exposure of the inhabitants was also assessed, as well as an estimation of the impact on health effects in comparison with the "normal" situation. In addition, an on-line perception questionnaire was disseminated during the lockdown period with the aim of gathering information on the way that the changes in sound environment were perceived by people all over France. More than 3000 respondents were collected and the main findings on sound perception are also reported in the paper.

The results of another study based on a on-line survey are reported by Bartalucci et al. [9]. Participants were asked to fill the questionnaire distributed to residents in the Italian territory, providing personal data, describing context and characteristics of the house in which they live and making a comparison of the lockdown soundscape with the pre-lockdown one. About 400 questionnaires have been collected and analyzed in order to define correlations between personal and perception variables. The outcomes of logistic regression models show that the perception of road traffic noise has increased for people over 35 years old and the neighborhood noise has been more statistically significant for employed respondents.

Spennemann et al. [10] point out that a side-effect of the dramatic social and behavioral change caused by governmental responses to COVID-19 has been a dramatic temporary alteration of urban cultural soundscapes. They consider the nature and extent of these soundscapes and explore to what extent the COVID-19-induced reality can be employed to project a future of urban cultural soundscapes if no active heritage intervention were to occur.

Sakagami [11] presents the results of a follow-up noise survey in a quiet residential area in Japan during the lockdown and a few weeks (June 2020) after the cancellation of the state of emergency. Since the results are almost the same, the author infers that either the noise level was reduced in June to a level that was almost the same as that during the state of emergency, or the noise level after its cancellation in May was possibly higher than usual. In either case, the change in noise level was small, and it was difficult to conclude which case was true.

The experience presented by Dance et al. [12] deals with engaging private companies, public organizations and academics to gather data in accessible places across the UK during the lockdown and subsequent periods. In addition, pre-existing on-going measurements from major infrastructure projects, airport, and planning applications were gathered to create the largest possible databank. A website was designed and developed to advertise the project, provide instructions and to formalize the uploading of noise data, observations and soundscape feedback. Two case studies gathered in the latter stage of full lockdown are presented in the paper to illustrate the changes in the environmental noise conditions relative to transport activity. 
Finally, Kalawapudi et al. [13] report the comparison of noise indices (LA10, LA50, LA90, noise pollution level LNP and noise climate NC) determined in 2020 during the Ganesh Chaturthi festival in Mumbai city, India, and those collected in the previous years of 2018 and 2019. The festival celebration in ecofriendly manner due to CoVID-19 restrictions led to significant decrease in noise levels, more than $25 \mathrm{~dB}(\mathrm{~A})$ in LAeq.

The Guest Editors are grateful to the all the Authors of the submitted papers and to the reviewers for their willingness to contribute to the success of the Special Issue.

It is worth to remind that the International Year of Sound (https://sound2020.org/) started in 2020 was extended to the end of 2021 and many events occurred and are still planned in such unique times to highlight the importance of sound and related sciences and technologies for all in society.

\section{References}

[1] Aletta F, Brinchi S, Carrese S, Gemma A, Guattari C, Mannini L, et al. Analysing urban traffic volumes and mapping noise emissions in Rome (Italy) in the context of containment measures for the COVID-19 disease. Noise Mapp. 2020;7:114-22.

[2] Alsina Pagès RM, Alías F, Bellucci P, Cartolano PP, Coppa I, Peruzzi A et al. Noise at the time of COVID 19: The impact in some areas in Rome and Milan, Italy. Noise Mapp. 2020;7:248-64.

[3] Bartalucci C, Borchi F, Carfagni M. Noise monitoring in Monza (Italy) during COVID-19 pandemic by means of the smart network of sensors developed in the LIFE MONZA project. Noise Mapp. 2020;7:199-211.

[4] Zambon G, Confalonieri C, Angelini F, Benocci R. Effects of COVID-19 outbreak on the sound environment of the city of Milan, Italy. Noise Mapp. 2021;8:116-28.

[5] Aletta F, Oberman T, Mitchell A, Tong H, Kang J. Assessing the changing urban sound environment during the COVID-19 lockdown period using short-term acoustic measurements. Noise Mapp. 2020:7;123-34. https://doi.org/10.1515/noise2020-0011.

[6] Vida Manzano J, Almagro Pastor JA, García Quesada R, Aletta F, Oberman T, A. Mitchell et al. The "sound of silence" in Granada during the COVID-19 lockdown. Noise Mapp. 2021;8:16-31.

[7] De Lauro E, Falanga M, Tedeschini Lalli L. The soundscape of the Trevi fountain in Covid-19 silence. Noise Mapp. 2020;7:212-22. https://doi.org/10.1515/noise-2020-0018.

[8] Munoz P, Vincent B, Domergue C, Gissinger V, Guillot S, Halbwachs Y et al. Lockdown during COVID-19 pandemic: impact on road traffic noise and on the perception of sound environment in France. Noise Mapp. 2020;7:287-302.

[9] Bartalucci C, Bellomini R, Luzzi S, Pulella P, Torelli G. A survey on the soundscape perception before and during the COVID-19 pandemic in Italy. Noise Mapp. 2021;8:65-88.

[10] Spennemann DHR, Parker M. Hitting the 'pause' button: What does COVID-19 tell us about the future of heritage sounds? Noise Mapp. 2020;7:265-75.

[11] Sakagami K. A note on the acoustic environment in a usually quiet residential area after the 'state of emergency' declaration due to COVID-19 pandemic in Japan was lifted: supplementary survey results in post-emergency situations. Noise Mapp. 2020;7:192-98.

[12] Dance S, McIntyre L. The Quiet Project - UK Acoustic Community's response to COVID19 during the easing of lockdown. Noise Mapp. 2021;8:32-40.

[13] Kalawapudi K, Singh T, Vijay R, Goyal N, Kumar R. Effects of COVID-19 pandemic on festival celebrations and noise pollution levels. Noise Mapp. 2021;8:89-93. 